\title{
Social Networks and the Convergence of Population Attributes: A Generalization
}

\author{
by \\ Jan K. Brueckner \\ Department of Economics \\ University of California, Irvine \\ 3151 Social Science Plaza \\ Irvine, CA 92697 \\ e-mail: jkbrueck@uci.edu \\ and \\ Oleg Smirnov \\ Departments of Economics and Geography \\ San Diego State University \\ San Diego, CA 92812 \\ e-mail: osmirnov@rohan.sdsu.edu
}

May 2006

\begin{abstract}
Analysis of social interactions has recently become an important area of economic research, and the focus of researchers in this area has increasingly shifted toward dynamic models. In one recent contribution, Brueckner and Smirnov (2006) analyze the evolution of population attributes in an exceedingly simple model where an agent's attributes at time $t$ are equal to the average attribute value among his acquaintances. The pattern of acquaintances in the population is determined by the social network, and Brueckner and Smirnov (BS) explore the effect of network characteristics on the convergence of population attributes over time. They show that some simple sufficient conditions on the network structure ensure convergence to a "melting-pot" equilibrium, where attributes are uniform across agents. The present paper provides a generalization of BS's analysis, allowing for a more general form of the rule governing the evolution of population attributes. The analysis shows that BS's previous conclusions continue to hold under this generalization, while also providing a result that can be applied more generally to other models.
\end{abstract}




\title{
Social Networks and the Convergence of Population Attributes: A Generalization
}

\author{
by
}

\author{
Jan K. Brueckner and Oleg Smirnov*
}

\section{Introduction}

Analysis of social interactions has recently become an important area of economic research, with the main contributions surveyed by Glaeser and Scheinkman (2002) and Scheinkman (2004). Increasingly, the focus of researchers in this area has shifted toward dynamic models, which explore the evolution of population attributes (sometimes portrayed as cultural assimilation) as agents interact through social networks. Examples include papers by Bisin and Verdier (2000, 2001), Bisin, Horst and Özgur (2006), Horst and Sheinkman (2002), Ioannides (2006) and Kónya (2002).

In a related contribution, Brueckner and Smirnov (2006) analyze the evolution of population attributes in an exceedingly simple model where an agent's attributes at time $t$ equal the average attribute value among his acquaintances. The pattern of acquaintances in the population is determined by the social network, and Brueckner and Smirnov (hereafter BS) explore the effect of network characteristics on the convergence of population attributes over time. They show that some simple sufficient conditions on the network structure ensure convergence to a "melting-pot" equilibrium, where attributes are uniform across agents.

The purpose of the present paper is to provide a generalization of BS's analysis, allowing for a more general form of the rule governing the evolution of population attributes. The analysis shows that BS's previous conclusions continue to hold under this generalization, while also providing a result that can be applied more generally to other models.

\section{Background}

In BS's model, the population consists of $m$ agents, and the attributes of agent $i$ at time $t$ are summarized by a scalar variable $\theta_{i}^{t}, i=1, \ldots, m$. If agent $i$ is acquainted with agent $j$, then 
$n_{i j}=1$, while if the agents are unacquainted, then $n_{i j}=0$ (symmetry of acquaintanceships implies $n_{i j}=n_{j i}$ ). The number of acquaintances for agent $i$ equals $q_{i}=\sum_{j=1}^{m} n_{i j}{ }^{1}$

The acquaintance pattern is summarized by the symmetric acquaintance matrix $N$, which has representative element $n_{i j} . \quad N$ is assumed to be irreducible, which means that any two agents are linked by a sequence of acquaintances, even though the agents may not be acquainted themselves. Formally, irreducibility means that, for all $(i, j), N_{i j}^{(k)}>0$ holds for some $k>0$, where this expression refers to element $(i, j)$ of the $k$ th power of the matrix $N$. Since $N_{i j}^{(k)}$ can be seen to equal the number of paths of length $k$ connecting agents $i$ and $j$ via other agents, the irreducibility requirement means that each pair of agents is connected by a path of some length.

In the basic version of BS's model, the evolution of population attributes is determined by a simple rule: agent $i$ 's attributes at time $t+1$ equal the average attributes at time $t$ among his acquaintances. The law of motion for attributes can thus be written

$$
\theta_{i}^{t+1}=\sum_{j=1}^{m}\left(\frac{n_{i j}}{q_{i}}\right) \theta_{j}^{t}
$$

To rewrite this law of motion in matrix form, let $A$ denote the (row-normalized) "acquaintance matrix", gotten by dividing each element of the $i$ th row of $N$ by the row sum $q_{i}$, for $i=1, \ldots, m$. Irreducibility of $N$ means that $A$ itself is irreducible. Recognizing that (2) can be written as $\theta_{i}^{t+1}=\sum_{j=1}^{m} a_{i j} \theta_{j}^{t}$, where the $a_{i j}$ are the elements of $A$, the law of motion can be rewritten in matrix form as

$$
\theta^{t+1}=A \theta^{t}
$$

where $\theta^{t+1}$ is the column vector $\left(\theta_{1}^{t+1} \theta_{2}^{t+1} \ldots \theta_{m}^{t+1}\right)^{\prime}$ and $\theta^{t}$ is defined analogously. Assuming that the evolutionary process for attributes starts at time zero, (2) yields

$$
\theta^{t}=A^{(t)} \theta^{0}
$$

where $\theta^{0}$ is the population attribute vector at time zero and $A^{(t)}$ denotes the $t$ th power of the acquaintance matrix. Initial attribute levels are assumed to be positive, so that $\theta^{0}>0$. 
BS explore the conditions under which $\theta^{t}$ converges to a vector with identical elements, which they term a "melting-pot" equilibrium given the resulting uniformity of population attributes. The key condition involves the "cyclicality" of the matrix $A$. Given its row normalization and irreducibility, the eigenvalues of $A$ (all of which are real) must lie in the interval $[-1,1]$, with the largest eigenvalue equal to 1 . If no -1 eigenvalue exists, then $A$ is said to be "noncylic." When $A$ has this property, standard results in linear algebra establish that $\theta^{t}$ converges to a melting-pot equilibrium. BS then ask what features of the social network (the acquaintanceships represented by $N$ ) yield noncyclicality of $A$, and they are able to offer two separate sufficient conditions. ${ }^{2}$

$\mathrm{BS}$ raise the question but did not fully address the more-general issue of attribute convergence in a model where the law of motion is itself time-dependent. In such a model, (2) is replaced by

$$
\theta^{t+1}=A^{t} \theta^{t}
$$

where the acquaintance matrix, which remains row-normalized, is now time-dependent (the $t$ superscript on $A$ represents time, not an exponent). Note that $A^{t}$ still reflects the structure of the social network, with the positive elements of $A^{t}$ and $N$ coinciding. When (4) is relevant, (3) is replaced by

$$
\theta^{t+1}=A^{t} A^{t-1} \cdots A^{1} A^{0} \theta^{0} .
$$

BS show that a law of motion like (4) arises when their basic model is generalized to allow endogenous intensity of interaction between acquaintances, as follows. Let $h_{i j}^{t}$ denote the time$t$ effort agent $i$ spends interacting with $j$, which must satisfy $\sum_{j=1}^{m} h_{i j}^{t}=1$, a fixed-total-effort constraint. Then, let the law of motion (1) be replaced by $\theta_{i}^{t+1}=\sum_{j=1}^{m} h_{i j}^{t} \theta_{j}^{t}$, so that the influence of an acquaintance's attributes depends on the intensity of interaction. To determine the chosen effort levels, let $f\left(h_{i j}^{t}\right) g_{i}\left(\theta_{j}^{t}\right)$ give the time- $t$ benefit that agent $i$ derives from interacting with acquaintance $j$, where $f$ gives effort productivity and $g_{i}$ relates the interaction benefit to $j$ 's characteristics. Agent $i$ allocates his effort to maximize $\sum_{j=1}^{m} n_{i j} f\left(h_{i j}^{t}\right) g_{i}\left(\theta_{j}^{t}\right)$ subject to the above constraint. For example, if $f\left(h_{i j}^{t}\right)=\ln \left(h_{i j}^{t}\right)$ and $g_{i}\left(\theta_{j}^{t}\right)=\theta_{j}^{t}$ (so that interactions with high- $\theta$ agents are preferred), then $h_{i j}^{t}=n_{i j} \theta_{j}^{t} /\left(\sum_{l=1}^{m} n_{i l} \theta_{l}^{t}\right)$, indicating that time- $t$ effort 
directed toward $j$ equals $j$ 's $\theta$-share among $i$ 's acquaintances at time $t$. The effort matrix at time $t$, which has elements $h_{i j}^{t}$, can then be written $H^{t} \equiv H\left(\theta^{t}, N\right)$, where the $t$ dependence arises because effort levels depend on the current attribute vector. The law of motion is then given by (4) with $H^{t}$ replacing $A^{t}$.

Although BS presented some suggestive numerical examples for this endogenous-interaction model, they left unanswered the general question of melting-pot convergence in a model with a time-dependent law of motion like (4). The purpose of the remaining analysis is to provide the missing result. It is shown that, if the law of motion is given by (4), and if the (rownormalized) acquaintance matrix $A^{t}$ is irreducible and noncyclic for all $t$, then melting-pot convergence occurs.

\section{Analysis}

To start the analysis, let the norm of a vector $x$, denoted $\|x\|$, be defined as the largest among the absolute values of its elements, so that $\|x\|=\max _{i}\left|x_{i}\right|$. Then, the following result can be established:

Lemma. Given row normalization of $A^{t}$, the norm of the attribute vector $\left\|\theta^{t}\right\|$ is a monotonically declining sequence, satisfying $\left\|\theta^{t+1}\right\| \leq\left\|\theta^{t}\right\|$. As a result, the attribute vectors $\theta^{t}$ converge in norm, i.e., there is a limit $c \geq 0$, such that $\lim _{t \rightarrow \infty}\left\|\theta^{t}\right\|=c$.

Proof: Let $k=\operatorname{argmax}_{i}\left\{\theta_{i}^{t}\right\}$, and $g=\theta_{k}^{t}$. Since $\theta_{j}^{t} \leq g$, for $j=1,2, \ldots, m$, it follows that

$$
\theta_{i}^{t+1}=\sum_{j} a_{i j}^{t} \theta_{j}^{t} \leq \sum_{j} a_{i j}^{t} g=g, \quad i=1,2, \ldots, m
$$

where the $a_{i j}^{t}$ are elements of $A^{t}$. Therefore, the norm of the attribute vector $\theta^{t+1}$ must be less than or equal to the norm in the previous time period: $\left\|\theta^{t+1}\right\| \leq\left\|\theta^{t}\right\|$. Convergence in norm then follows from observing that a monotonically declining sequence bounded from below has a limit (the norm is bounded below by zero).

To establish the main result, note that for an irreducible, noncylic matrix, there is an integer power $p>0$ such that $A^{(p)}>0$, indicating that all elements of the matrix $A^{(p)}$ are positive (see Bapat, Raghavan (1997), pp. 40-41). Along with the lemma, this fact then yields 
Proposition 1. If the (row-normalized) acquaintance matrices $A^{t}$ are irreducible and noncyclic for all $t$, and the law of motion is given by (4), then the population attribute vector $\theta^{t}$ converges to a melting-pot equilibrium.

Proof: Assume that convergence in norm has occurred by some time period $T_{c}$, so that $\mid\left\|\theta^{t}\right\|-$ $c \mid<\epsilon$, for all $t>T_{c}$, where $\epsilon>0$ is an arbitrarily chosen positive value. Select a point in time $T=T_{c}+p$ such that $N^{p}>0$; the existence and finiteness of this integer $p$ is guaranteed by the above property of irreducible, noncyclic matrices.

Since the matrices $A^{T-1}, A^{T-2}, \ldots, A^{T-p}$ have the same structure of nonzero elements as $N$ and $N^{p}>0$, the matrix $B$ obtained as the product of matrices $A^{T-1}, A^{T-2}, \ldots, A^{T-p}$, is positive: $B=A^{T-1} A^{T-2} \ldots A^{T-p}>0$. Moreover, since the matrix $B$ is the product of row-normalized matrices, it is also row-normalized: $b_{i j}>0$ holds for all $i, j$, and $\sum_{j} b_{i j}=1$, $i=1,2, \ldots, m$, where the $b_{i j}$ are elements of $B$.

Convergence in norm implies ||$\left|\theta^{T} \|-c\right|<\epsilon$ and thus that $\left|\theta_{i}^{T}-c\right|<\epsilon$ holds for each agent $i$. Since $\theta_{i}^{T}=\sum_{j} b_{i j} \theta_{j}^{T-p}$, convergence in norm then implies

$$
\left|\sum_{j} b_{i j} \theta_{j}^{T-p}-c\right|<\epsilon
$$

Next, observe that, for $(7)$ to be satisfied, $-\left(\sum_{j} b_{i j} \theta_{j}^{T-p}-c\right)<\epsilon$ must hold. Letting $\theta_{s}^{T-p}$ denote the smallest element in the attribute vector $\theta^{T-p}$, rerrangement of the last inequality then yields

$$
c-\epsilon-\sum_{j \neq s} b_{i j} \theta_{j}^{T-p}<b_{i s} \theta_{s}^{T-p} .
$$

Since $\theta_{j}^{T-p}<c+\epsilon$ must hold and all the $b_{i j}$ are positive, (8) implies

$$
c-\epsilon-\sum_{j \neq s} b_{i j}(c+\epsilon)<b_{i s} \theta_{s}^{T-p} .
$$

But using $\sum_{j} b_{i j}=1$, the left-hand side of (9) equals $c b_{i s}+\left(b_{i s}-2\right) \epsilon$. Dividing both sides by the positive value $b_{i s}$ then yields

$$
c+\left(1-2 / b_{i s}\right) \epsilon<\theta_{s}^{T-p} .
$$


Since this inequality is satisfied for any arbitrary $\epsilon$, and since $\theta_{s}^{T-p} \leq \theta_{j}^{T-p}<c+\epsilon$, for $j \neq s$, if follows that all the $\theta_{i}^{T-p}, i=1,2, \ldots, m$, converge to $c .{ }^{3}$ This fact establishes convergence of $\theta^{t}$ to a vector with all components equal to $c$, a melting-pot equilibrium.

\section{Conclusion}

Proposition 1 shows that melting-pot convergence occurs in BS's endogenous-interaction model, provided that the underlying acquaintance matrix $N$ is irreducible and noncyclic. This conclusion follows because the matrix $H^{t}$ in the model's law of motion inherits these properties of $N$. Therefore, BS's sufficient conditions for noncylicality of $N$ yield melting-pot convergence both in their basic model and in the endogenous-interaction case.

Proposition 1 has more-general implications, however. It establishes that for any timedependent linear rule governing the evolution of attributes within a social network, meltingpot convergence occurs provided that the underlying $N$ matrix is irreducible and noncyclic. A randomized rule, for example, where one acquaintance chosen at random in each period receives a weight of $2 / 3$ in (1), with the remaining acquaintances receiving weights adding to $1 / 3$, would yield melting-pot convergence. Such a rule could reflect a type of interaction where people are most influenced by the "last person they've talked to." 


\section{References}

Bapat, R.B., Raghavan, T.E.S., 1997. Nonnegative Matrices and Applications, Cambridge University Press, Cambridge.

Bisin, A., Verdier, T., 2001. The economics of cultural transmission and the dynamics of preferences, Journal of Economic Theory 97, 298-319.

Bisin, A. AND Verdier, T. 2000. Beyond the melting pot: Cultural transmission, marriage, and the evolution of ethnic and religious traits, Quarterly Journal of Economics 115, 955988.

Bisin, A., Horst, U., Özgur, O., 2006. Rational expectations equilibria of economies with local interactions, Journal of Economic Theory 127, 1-43.

Brock, W.A., Durlauf, S.N., 2001. Discrete choice with social interactions, Review of Economic Studies 68, 235-260.

Brueckner, J.K., Smirnov, O.A., 2006. Workings of the melting pot: social networks and the evolution of population attributes, Journal of Regional Science, forthcoming.

Glaeser, E., Scheinkman, J.A., 2002. Non-market interactions, I:n L. Dewatripont, P. Hansen and S. Turnovsky (Eds.), Advances in Economics and Econometrics: Theory and Applications, Cambridge, Cambridge University Press.

Horst, U., Scheinkman, J.A., 2005. Equilibria in systems of social interactions, Journal of Economic Theory, forthcoming.

IoAnnides, Y.M., 2006. Topologies of social interactions, Economic Theory 28, 559-584.

KÓNYA, I., 2002. A dynamic model of cultural assimilation, Unpublished paper, Boston College.

Scheinkman, J.A., 2004. Social interactions, In: L.H. Goulder and W.A. Pizer (Eds.), New Palgrave Dictionary of Economics, 2nd Edition, New York, Macmillan, forthcoming. 


\section{Footnotes}

${ }^{1}$ It might appear that $n_{i i}$ should always equal 1 . However, the case where $n_{i i}=0$ is possible and important, and it is discussed in foonote 2 below.

${ }^{2}$ One sufficient condition is that at least one agent $i$ is "self-referential," with $n_{i i}=1$. Then, the agent's time- $(t+1)$ attributes depend partly on his attributes at time $t$ (see (1)). If this condition does not hold, then all agents are pure mimickers, with $\theta_{i}^{t+1}$ for each agent $i$ depending only on the $\theta_{j}^{t}$ values of his acquaintances. The second sufficient condition is that there exist two agents who are jointly acquainted with a third agent. As can be seen, these sufficient conditions are quite weak.

${ }^{3}$ Note that while $b_{i s}$ depends on $T$, it is independent of $\epsilon$. 